\title{
Analysis of the light and radial velocity curves of transiting extra-solar planets
}

\author{
Alvaro Giménez \\ Centro de Astrobiologia, CSIC-INTA, Madrid \\ email: agimenez@rssd.esa.int
}

\begin{abstract}
Several approaches have been followed for the analysis of the light curves of transiting extra-solar planets. From simple approximate algorithms and synthetic models to semi-analytical complete solutions. The different alternatives are discussed with their strengths and weaknesses. Analytical expressions can also provide a deeper insight into the main system parameters from the measurement of a few strategic points describing the shape of the light curve.
\end{abstract}

\section{Introduction}

It is well known, from decades of experience in the analysis of the light curves of eclipsing binaries, that the periodic transits of extra-solar planets in front of their host stars should provide the necessary information to derive the inclination of their orbit and the relative radii of both the star and the planet. In fact, non-distorted components should allow for a good determination of the sum of the relative radii $r_{s}+r_{p}$ from the total duration of the eclipse and, since transits are equivalent to annular eclipses produced by an opaque dark disk, a good estimation of the ratio of radii $k=r_{p} / r_{s}$ is also possible. On the other hand, the expected small values of $k$ for planetary transits imply that a linear limb-darkening law is generally not a good approximation and, at least, a second order law is required.

The mathematical problem of calculating the loss of light due to a transit can be expressed as the integration of the overlapping area (the occultation of the stellar surface by the planet), in such a way that the total light $L$, at any time $t$, is given by

$$
l(t)=L_{s}+L_{p}-\alpha(t) L_{s}=1-\alpha L_{s}
$$

where the subindex $s$ stands for the star and $p$ for the planet. The function $\alpha$ is the relative loss of light that can also be expressed by the integral,

$$
\alpha=\int_{S} I \cos \gamma d \sigma
$$

over the overlapping area $S$, where $\gamma$ denotes the foreshortening angle and $\sigma$ the surface element. The distribution of light over the stellar surface $I(\mu)$, where $\mu=\cos \gamma$, takes into account the effect of limb darkening and can be expressed by means of, for example, a quadratic law in the form,

$$
I(\mu)=I(1)\left[1-u_{a}(1-\mu)-u_{b}(1-\mu)^{2}\right]
$$

where $I(1)$ is the radiation intensity at the centre of the disk, while $u_{a}$ and $u_{b}$ are the stellar limb-darkening coefficients, available in tabulated form as derived from different model atmospheres for a variety of effective temperatures, surface gravities and wavelength ranges. 
The issue now is the computation of the function $\alpha$ in terms of the orbital phase and the elements of the system that we want to solve for. There are basically three methods to calculate $\alpha$ : one based on simple approximated algorithms, another one on synthetic light curve models, and a third one based on the analytical solution of the corresponding integrals.

\section{Methods and precisions}

The precision required by the model computations depends of course on how accurate the observed light curves are. For a Jupiter-size planet, the depth of the photometric transit is of the order of $1 \%$ and the precision required for the light curve is around $0.1 \%$ in order to determine the geometrical elements, or around $0.01 \%$ to also obtain the limb darkening coefficients. For the analysis of these light curves, the computation of the $\alpha$ functions should be better than $\approx 10^{-5}$. In the case of Earth-like planets, the challenge is much greater since the previous parameters are expected to be smaller by two orders of magnitude and therefore observed light curves have to be better than $\approx 10^{-5}$, even assuming the limb-darkening coefficients, while the predicted model light curves must reach at least a precision of $10^{-6}$.

Out of the three possible solutions for the computation of $\alpha$ mentioned above, approximated algorithms represent the less accurate approach. Approximate algorithms have been used by Deeg et al. (2001), extended to non-linear limb-darkening by Mandel \& Agol (2002) and analysing the effects of the assumptions by Seager \& Mallén-Ornelas (2003). They are recommended for a quick estimation of the transit parameters in the case of circular orbits and are based on the assumption that the relative radius of the planet $r_{p}$ is smaller than the variation of the limb-darkening function. The precision of these procedures is generally not good enough for a detailed analysis of the currently available high-quality light curves.

Another option is to use synthetic models where the integration of the $\alpha$ function is done through numerical summation of, either a distributed array of points over the stellar surface, or concentric quasi-circular elliptical rings of finite size. Synthetic models allow for the possibility to take into account the eventual non-sphericity of the components and other more subtle effects in the host star's radiation pattern. It has been found that the EBOP code, as described by Etzel (1993), and based on the model by Nelson \& Davis (1972), is the best suited for the analysis of the light curves of extrasolar planetary transits. In this case, the Roche geometry does not need to be taken into account and the code can easily be adapted to non-linear limb-darkening laws (Giménez \& Diaz-Cordovés, 1993). Of course, limb darkening has to be assumed to be constant within pre-defined rings and the end precision is driven by the relative size of the planet with respect to the width of the rings. Precisions are thus limited to $\approx 10^{-5}$, which is certainly enough for currently available data but possibly not for the analysis of Earth-like planetary transits as expected from future space missions. On the other hand the use of the differential correction optimization process contemplated in EBOP is only applicable in the case of light curves better than around $1 \%$ of the depth of the transit.

As a third option, an analytical solution of the $\alpha$ function can be obtained in the case of circular disks, valid for any degree of limb-darkening and any adopted value of the orbital eccentricity. Two approaches are possible here: a direct integration (by hand) leading to complex but accurate expressions that can be easily coded (Mandel \& Agol, 2002), or a solution based on the cross correlation of two optical apertures (Kopal, 1979) as given by Giménez (2006a). 


\section{Advantages of the analytical solution}

In the case of an analytical solution as described by Giménez (2006a), the involved $\alpha$ functions can be expressed in a closed form, valid for any degree of limb darkening, whose computation can be very easily programmed. Codes for the calculation of these functions are provided via ftp at http://thor.ieec.uab.es/LRVCODE/ where not only the equations for the analysis of the light curves can be found but also those for the computation of the related Rossiter-McLaughlin effect in the radial velocity curve during transit phases (see also Giménez 2007). Indeed, the equations used in the computation of the $\alpha$ functions of the light curves of transiting planets are also involved in the calculation of the mentioned Rossiter-McLaughlin effect, as described by Giménez (2006b), by means of the first derivative of the $\alpha$ functions. There is no need for additional assumptions or a complicated integration by hand, as performed by Ohta et al. (2005). Furthermore, a simultaneous solution of the light and radial velocity curves is possible with the proposed equations leading to a better understanding of the involved parameters.

By using the equations of the direct analytical solution, the achievable precision is not limited by the relative size of the planet with respect to the limb-darkening variations. It is controlled via the number of terms in the involved Jacobi polynomials, N (with standard values between 2000 and 3000). In the case of the light curve of the prototypical extrasolar planet around HD 209458, the use of $N=2000$ provides a light curve with a precision at any phase better than $10^{-8}$, well beyond the observational limitations.

On the other hand, an analytical solution allows for specific cases in the light curve to be solved. For example, using the depth of the transit at its central phase $\Delta_{0}$, the ratio of radii $k$ can be easily shown to be closely approximated by,

$$
k=0.005+\sqrt{1-\left(1-1.263 \Delta_{0}\right)^{2 / 3}}
$$

as well as the range between maximum $k=\sqrt{1-\Delta_{0}}$ and minimum values,

$$
k=\sqrt{1-[1-(1-\Delta)]^{2 / 3}} .
$$

On the other hand, the orbital inclination can also be estimated from the duration of the transit $T$ if the stellar relative radius is known, since,

$$
i=\arcsin \sqrt{\frac{1-r_{s}^{2}(1+k)^{2}}{1-(\pi T / P)^{2}}}
$$

while, if the stellar density $\rho$ can be evaluated,

$$
r_{s}=0.238 \rho^{-1 / 3} P^{-2 / 3}
$$

Finally, an estimation of the orbital inclination is also possible from a detailed inspection of the shape of the light curve, with no previous knowledge of the size of the star. The solution of the $\alpha$ function for the internal tangency point, i.e., at $\delta=r_{s}-r_{p}$ can be explicitly written, independent of the orbital inclination, in such a way that the depth of the light curve at such point $\Delta_{1}$ is given by,

$$
\Delta_{1}=1+0.015 k-0.89 k^{2}-0.61 k^{3}
$$

as a good approximation for solar-type host stars. Thus the phase $\theta_{1}$ of the internal tangency point can be easily measured from the light curve and, denoting similarly the first contact or beginning of the transit phase $\theta_{2}$ corresponding to $\delta=r_{s}+r_{p}$ 
for $\alpha=0$,

$$
\frac{1}{r_{s}}=(1+k)^{2}-4 k /\left[1-\frac{\cos ^{2} \theta_{1}}{\cos ^{2} \theta_{2}}\right]
$$

from which the orbital inclination can again be derived using (3.3).

\section{References}

Deeg, H. J, Garrido, R., \& Claret, A. 2001, New Astron., 6, 51

Etzel, P. B. 1993. In "Light curve modeling of eclipsing binary stars" (Ed. E. F. Milone), p. 113, Springer Verlag, New York.

Giménez, A. 2006a, Astron. Astrophys., 450, 1231 (Paper I)

Giménez, A. 2006b, ApJ, 650, 408 (Paper II)

Giménez, A. 2007, Astron. Astrophys., 474, 1049

Giménez, A. \& Diaz-Cordovés, J. 1993. In "Light curve modeling of eclipsing binary stars" (Ed. E.F. Milone), p. 125, Springer Verlag, New York.

Kopal, Z. 1979, Language of the Stars (Reidel Publ., Dordrecht, Holland).

Mandel, K. \& Agol, E. 2002, ApJ, 580, L171

Nelson, B. \& Davis, W. D. 1972, ApJ, 174,617

Ohta, Y., Taruya, A., \& Suto, Y. 2005, ApJ, 622, 1118

Seager, S. \& Mallén-Ornelas, G. 2003, ApJ, 585, 1038 